Referencia para citar este artículo: Fernández-Martínez, A. M. \& Montero-García, I. (2016). Aportes para la educación de la Inteligencia Emocional desde la Educación Infantil. Revista Latinoamericana de Ciencias Sociales, Niñez y Juventud, 14 (1), pp. 53-66.

\title{
Aportes para la educación de la Inteligencia Emocional desde la Educación Infantil*
}

\author{
Ana María Fernández-Martínez ${ }^{* *}$ \\ Maestra colegio Juan XXIII de la Chana, Granada, España. \\ INMACULADA MoNTERo-GARCÍA ${ }^{* * *}$ \\ Profesora Universidad de Granada, España.
}

\section{Artículo recibido en noviembre 24 de 2014; artículo aceptado en abril 12 de 2015 (Eds.)}

- Resumen (descriptivo): La labor de los maestros y maestras no es solo la trasmisión de conocimientos académicos, sino la de guiar a nuestros alumnos y alumnas para que vivan la experiencia del aprendizaje para la vida de manera enriquecedora e innovadora. De esta forma, garantizamos que afronten los retos con mayor comprensión, creatividad y sentido de la responsabilidad. La educación emocional puede ser un importante instrumento para este logro. Sin embargo, durante mucho tiempo no se ha trabajado en las escuelas porque se consideraba que entorpecía el desarrollo cognitivo. Gracias a los resultados de numerosos estudios, en la actualidad sabemos que no solo emoción y cognición son compatibles, sino que además son inseparables y se benefician mutuamente a través de las relaciones circulares existentes entre ellas. Propondremos finalmente algunas actuaciones que, desde la educación infantil, contribuyen a propiciar dicho proceso.

Palabras clave: Inteligencia emocional, educación infantil, desarrollo emocional (Tesauro Eric), inteligencia, cognición (Tesauro de Ciencias Sociales de la Unesco).

\section{Contributions to emotional intelligence education from early childhood education}

- Abstract (descriptive): A teacher's task is not just the transmission of academic knowledge but also involves guiding students so that they have rich and innovative learning experiences throughout their lives. In this way teachers guarantee that students will be able to tackle challenges with better understanding, creativity and sense of responsibility. Emotional intelligence education can be an important tool in achieving this aim. However, for a long time emotional intelligence education has not been used by schools because it was considered that it hindered cognitive development. Thanks to the results of many studies we now know that emotion and cognition are not only compatible but are also inseparable and mutually beneficial to each other through the circular relationships that exist between them. Finally, the authors propose some actions from early childhood education that facilitate the teaching of emotional intelligence.

Key words: Emotional intelligence, early childhood education, emotional development (Eric Thesaurus), intelligence, cognition (Unesco Social Sciences Thesaurus).

\footnotetext{
* Este artículo de reflexión parte del estudio "Importancia del desarrollo de la Inteligencia Emocional en la Educación Infantil”, realizado para obtener el Trabajo Fin de Grado de la titulación de Grado de Maestro de Educación Infantil en la Universidad de Granada (España), Facultad de Ciencias de la Educación. Realizado entre Marzo del 2014 y Junio del 2014. Área: Ciencias de la Educación. Subárea: Educación general.

**_Maestra de Educación Infantil. Universidad de Granada (España). Correo electrónico: anamaria_92_@hotmail.com

*** Doctora en Pedagogía. Profesora del Departamento de Pedagogía, Universidad de Granada (España). Correo electrónico: imontero@ugr.es
} 


\section{Aportes para a educação da inteligência emocional a partir da educação infantil}

- Resumo (descritivo): O trabalho dos professores e das professoras não é apenas a transmissão de conhecimentos acadêmicos, mas também a orientação aos alunos e às alunas para que tenham uma experiência de aprendizagem para a vida, de uma forma enriquecedora e inovadora. Desse modo, garantimos que os alunos serão capazes de enfrentar os desafios com maior compreensão, criatividade e sentido de responsabilidade. A educação emocional pode ser uma ferramenta importante para atingir esse objetivo. No entanto, durante muito tempo, não tem funcionado nas escolas, por ter sido considerada como uma dificuldade para o desenvolvimento cognitivo. Graças aos resultados de vários estudos, atualmente sabemos que a emoção e a cognição não são apenas compatíveis, mas também são inseparáveis e mutuamente benéficas por meio das relações circulares que existem entre elas. Por fim, propomos alguns procedimentos que, desde a educação infantil, contribuem para facilitar esse processo.

Palavras-chave: Inteligência emocional, educação infantil, desenvolvimento emocional (Eric Thesaurus), inteligência, cognição (Tesauro de Ciências Sociais da Unesco).

-1. Introducción. -2. Evolución y actualidad de la inteligencia emocional como constructo. -3. Trascendencia de la Inteligencia Emocional en el ámbito educativo. Consideraciones desde la Educación Infantil. -4. Estrategias para el desarrollo de la I.E. en el aula. -5. Conclusiones y propuestas educativas. -Lista de referencias.

\section{Introducción}

La pretensión de este trabajo es dar a conocer la importancia que tiene el desarrollo de la I.E. (Inteligencia Emocional) en la vida de las personas así como la necesidad de potenciar su educación, especialmente en el segundo ciclo de Educación Infantil. Sabiendo que es este un periodo crítico, se parte de la hipótesis de que dicha educación influirá positivamente en el desarrollo de la inteligencia cognitiva y el éxito en los diferentes ámbitos de la vida.

La labor y responsabilidad de los maestros no es solo enseñar conocimientos a los alumnos y alumnas, sino contribuir al perfeccionamiento de cada uno de estos niños y, para lograr este objetivo, la I.E. puede ser una buena herramienta. De este modo, fomentando su desarrollo, es posible contribuir a la construcción de un mundo más inteligente y más feliz. A pesar de que la I.E. se está convirtiendo en un tema muy popular, desde el punto de vista de la educación aún no se le concede todo el reconocimiento que merece. Porque no sólo es preciso justificar la trascendencia de la educación emocional para niños y niñas. Conviene tener en cuenta que padres, maestros, monitores, educadores... también requieren pasar del convencimiento a la adquisición de destrezas para facilitar y potenciar el desarrollo de las emociones (López-Cassà, 2005).

Las consecuencias que se derivan de estos presupuestos son varias. En primer lugar, es preciso seguir trabajando el tema de la I.E. en nuestro país. Es cierto que en España es cada vez mayor el número de estudios y aportaciones a la comunidad científica. Hoy en día se está comenzando a convertir en un tema de interés, sin embargo aún quedan muchos retos que afrontar en un futuro inmediato (Pena \& Reppeto, 2008), ya que la mayor parte de las investigaciones y estudios que poseemos proceden de otros países.

En segundo lugar, relacionado con lo anterior, hay mucha gente que posee una idea errónea de lo que significa la I.E., pues a pesar de conocer el término, desconocen lo que implica, sus repercusiones, o su compatibilidad con otras inteligencias. Por ello, es conveniente esclarecer todas estas dudas generales y minimizar las confusiones en la concepción del constructo.

En tercer lugar, a esta fundamentación teórica sobre la I.E. le sigue un quehacer práctico en los colegios, fundamental para su completo desarrollo. Sin embargo, debido al desconocimiento que existe con frecuencia no se lleva a cabo y privamos de este modo a 
toda la comunidad educativa de sus aportes. Es esencial dar este tema a conocer, sobre todo a quienes están involucrados en su educación.

En cuarto lugar, y para finalizar, existen pocos escritos que puedan ser utilizados como guías que contengan orientaciones concretas sobre cómo actuar en un aula de segundo ciclo de Educación Infantil, de modo que la I.E. no quede en un mero planteamiento teórico. En esta línea, más adelante ofreceremos algunas pautas para lograr una aplicación práctica que favorezca una adecuada educación emocional.

Teniendo en cuenta estas consideraciones, se exponen los objetivos a alcanzar: a) Fundamentar la importancia de la I.E. en Educación Infantil; b) Explicar la compatibilidad e interrelaciones existentes entre emoción y cognición; c) Analizar algunos de los factores del desarrollo de la I.E. que más influyen en el aprendizaje y bienestar de los niños; d) Diseñar pautas concretas de actuación sobre cómo desarrollar la I.E. en Educación Infantil; e) Proponer alternativas desde el ámbito profesional para que la I.E. pueda ser llevada a la práctica en Educación Infantil.

\section{Evolución y actualidad de la inteligencia emocional como constructo}

La inteligencia no es solo un conjunto de aptitudes que se miden por un test, tal y como coinciden en afirmar desde hace unos años psicólogos y educadores (Gardner, 1993, Goleman, 1998, Bisquerra, 2003, Marrodán, 2013), sino que constituye una capacidad muy genérica que engloba a su vez distintas capacidades cognitivas. Dichas capacidades pueden llegar a relacionarse incluso con la dimensión emocional, afectiva y social, a la que hoy en día también conocemos como "Inteligencia Emocional" (González-Ramírez, 2007).

Según Goleman (1995, pp. 80-81) la I.E. "abarca cinco competencias principales: el conocimiento de las propias emociones, la capacidad de controlar las emociones, la capacidad de motivarse a uno mismo, el reconocimiento de las emociones ajenas y el control de las relaciones".
Fue este investigador, Daniel Goleman, psicólogo, filósofo y periodista, quien popularizó el concepto de I.E. tras el éxito de su "best-seller" mundial "Inteligencia Emocional" en 1995. Desde entonces, ha sido uno de los constructos más difundidos e investigados, convirtiéndose en un tema de gran interés social y científico.

Sin embargo, no fue este autor el primero en hacer referencia a la I.E. Las primeras raíces del término nos trasladan a 1859, cuando Charles Darwin publicó su teoría y consideró la importancia de la expresión emocional para la supervivencia y adaptación de los seres vivos con el medio ambiente (Rodríguez-Sánchez, 2000).

Debido a la aparición del conductismo en 1912, en los años siguientes se frenó el estudio de los procedimientos no observables (Mestre \& Fernández-Berrocal, 2007), y la inteligencia y las emociones dejaron de ser objeto de interés. Hasta que no se debilitaron las posturas conductistas, no volvió a resurgir el deseo por conocer los procesos cognitivos. Encontramos entonces otra de las primeras ideas relacionadas con la I.E. que nos remonta al año 1920, ya que Thorndike ya anunció que la inteligencia social era la habilidad para poder entender a las personas y cooperar con ellas (Birknerová, Frankovský \& Zbihlejová, 2013). Más tarde, en 1940, Wechsler defendió la influencia de los aspectos no cognitivos en la inteligencia de las personas (Dumbrava, 2011).

En los años noventa el concepto de inteligencia adquirió una dimensión más amplia y realista. Entonces surgieron conceptos como el de las inteligencias múltiples, en el que Edward Gardner (1993) aconsejaba apreciar a aquellos individuos con diversos estilos de aprendizaje y potenciales e introdujo la inteligencia interpersonal.

No obstante, quienes definieron por primera vez el concepto de I.E. como tal fueron los trabajos de Salovey y Mayer (1990) y Mayer, Dipaolo y Salovey (1990). En el primero afirmaron que la I.E. es "la capacidad de entender y controlar las emociones propias y ajenas, discriminar entre ellas y utilizar esta información para guiar el pensamiento y las acciones de uno" (p. 189). En el segundo 
demostraron empíricamente cómo la cognición y la emoción pueden vincularse para realizar complejos procesamientos de la información.

Aun así, como indicábamos anteriormente, no fue hasta 1995 cuando Daniel Goleman popularizó este concepto. Desde entonces, el constructo de I.E. ha evolucionado de forma indudable $\mathrm{y}$ actualmente no se considera el sistema emocional como un elemento perturbador de la inteligencia (Mestre \& Fernández-Berrocal, 2007), sino como potenciadora de la misma. Hoy en día, la I.E. es un tema de gran actualidad, convirtiéndose en objeto de interés y debate para muchos científicos, psicólogos y educadores.

En el contexto latinoamericano, Castro, Van der Veer, Burgos-Troncoso, Meneses-Pizarro, Pumarino-Cuevas y Tello-Viorklumds (2013) han publicado recientemente un interesante estudio acerca de la educación emocional, donde describen las teorías subjetivas presentes en los libros con consejos para padres y madres con hijos en la primera infancia. En este artículo se reconoce que apenas se han realizado análisis de este tipo de libros en Latinoamérica.

Por su parte, desde Argentina, la profesora Mikulic (2013), reconoce que en este país, como en otros muchos, tradicionalmente las emociones poco se han tenido en cuenta como procesos relevantes del desarrollo, de forma concreta en el espacio educativo, "donde los aspectos intelectuales y cognitivos han absorbido, casi de manera exclusiva, toda la atención" (p. 33). Esto se considera especialmente importante en un contexto donde la exclusión y los déficits educacionales del nivel primario escolar aparecen como problemas estructurales, y la inequidad social se revela como uno de los factores subsistentes que posee a su vez claros efectos regresivos sobre los niños, especialmente sobre los más vulnerables. Sin embargo, o quizá por ello, del mismo modo se advierte de manera notable cómo en las últimas décadas se comienza a detectar el incipiente interés de quienes comienzan a pensar en términos de educación emocional y social. En este sentido, también desde Argentina, otros estudios han mostrado la necesidad de concebir la construcción del conocimiento como proceso, en el que los niños se tornan activos y donde la articulación dialéctica entre las etapas de desarrollo de la infancia contribuyen a que podamos pensar en ellos como sujetos complejos, capaces de asociar, discutir, usar, apropiarse de lo que oyen, de lo que ven, representar modelos ausentes, en suma, permanecer dinámicos en la propia diversificación de intereses, tareas, roles y elecciones (Duer, 2010).

En el contexto colombiano, una reciente tesis doctoral avala la idea acerca de que, aun reconociendo avances importantes en el campo de la inteligencia emocional, aún queda mucho por investigar en este ámbito desde el ser humano, su interioridad y su interacción empática con los demás (Buitrago, 2012). Llevándolo al terreno de la Educación Infantil, es otra tesis la que puntualiza cómo en Colombia, la Política Nacional de primera infancia hace referencia a la generación de escenarios institucionales, familiares, comunitarios, para facilitar la participación en prácticas sociales y culturales que favorezcan, por medio del juego y el arte, el desarrollo del lenguaje, la creatividad, la expresión y el sentido estético (Durán, 2013), elementos presentes en la práctica de la educación emocional.

Así pues, de manera global la emergencia, oportunidad y uso del término en distintas esferas y países, han convertido este constructo en algo muy novedoso, de forma que hay quienes lo refieren como un tema de "moda" (Marrodán, 2013). Desde nuestra experiencia y formación, creemos firmemente en lo desacertado de esta expresión. No obstante, es preciso reconocer que es un concepto que está teniendo una rápida expansión, con una progresión evidente, lo cual no siempre facilita el consenso y el reconocimiento exacto acerca de lo que de manera efectiva puede llegar a significar en los distintos ámbitos o aspectos a los que pudiera transferirse. Una de las esferas donde esta inteligencia es más conocida y donde cobra especial relevancia, es en el mundo laboral y en las empresas, ya que, tal y como Goleman (1995) predijo, las personas más exitosas no solo a nivel personal, sino también a nivel laboral son aquellas que tienen su I.E. más desarrollada. Lo cual nos hace replantearnos por qué no comienza a trabajarse 
de manera intencional desde la Educación Infantil, cuando es esta la edad evolutiva que podría proporcionar el momento óptimo para su desarrollo.

\section{Trascendencia de la inteligencia emocional en el ámbito educativo. Consideraciones desde la Educación Infantil}

De acuerdo con Boix (2007), el hecho de que la clase, el colegio o la vida de las personas sea un infierno o un paraíso depende de las emociones que se vivan allí; y es que si hay algo por lo que realmente merece la pena desarrollar nuestra I.E. es porque ésta es un factor fundamental en nuestra realización como personas y nuestra felicidad personal, objetivo principal en la vida de cualquier ser humano. Valero (2009) lo expresa del siguiente modo: "La educación de los sentimientos es garantía de una vida feliz" (p. 58). Además, según GonzálezRamírez (2007), el mundo de los sentimientos y de las emociones nos permite adaptarnos mejor al mundo social, tener una comunicación eficaz, motivación personal, lograr objetivos, resolver conflictos interpersonales, ser empáticos... Por tanto, el éxito no depende exclusivamente del cociente intelectual, la I.E. juega un papel indudable (Goleman, 1998). Incluso podríamos decir que la educación emocional es aquella que nos permite formar personas más responsables, ya que nuestras vidas no están solamente gobernadas por la lógica, sino que nuestro mundo emocional motiva y mueve nuestras decisiones y acciones (Freshwater \& Stickley, 2004).

Tanto es así que, según Bisquerra (2003), el desarrollo de la I.E. no solo garantiza el bienestar personal, sino que hace menos probable que la persona se implique en comportamientos de riesgo como los conflictos, la violencia, el estrés, la depresión o el consumo de drogas. Razón de más para evidenciar la importancia de fomentar la educación emocional desde la escuela. Y es que, además de ser adecuada para aquellos alumnos que tienen problemas, presentan dificultad o por sus circunstancias tienen más probabilidad de iniciarse en comportamientos de riesgo, es beneficiosa para todos y cada uno de ellos, con independencia de las características personales, etnia, cultura o sexo, puesto que abarca a la persona en su conjunto de manera integral (Payton et al., 2008). Su carácter teleológico tiene que ver con los valores que conducen el proceso educativo. En este sentido, convenimos con el profesor Marina que "la educación emocional es un saber instrumental que ha de encuadrarse en un marco ético que le indique los fines, y debe prolongarse en una educación de las virtudes que permita realizar los valores fundamentales" (Marina, 2005, p. 27).

Ante lo expuesto cabe preguntarse cómo se adquiere la I.E; si viene dada o si es posible desarrollarla con el paso de los años. Con respecto a estas cuestiones hay cierto consenso entre los diferentes autores.

Según Goleman (1998, p. 21) "el grado de inteligencia emocional no está determinado genéticamente". Este autor señala que es posible ir aprendiendo a ser más intelectuales emocionalmente a medida que vamos teniendo distintas experiencias. Gallego-Gil y GallegoAlarcón (2006), sí que opinan que la I.E. es un potencial con el que se nace, pero coinciden en que es posible seguir desarrollándolo a través de la educación. Mestre \& Fernández-Berrocal (2007, p. 41) comparten con estos últimos autores las dos ideas, puesto que señalan que "la inteligencia emocional puede ser mejorada a través de la educación"; no obstante, la base de la que cada niño parte es diferente y ello hace que el aprendizaje emocional no sea el mismo para todos. Ahora bien, en este punto, ¿dónde aprendemos a desarrollar nuestra I.E.?

El primer lugar donde el niño desarrolla su I.E. es en el seno familiar, ya que no se trata de un simple aprendizaje cognoscitivo, sino que está lleno de emocionalidad al producirse en un ambiente tan afectivo como es el hogar (Gallego-Gil \& Gallego-Alarcón, 2006). Aunque es posible tener intensas experiencias afectivas fuera de este ámbito que beneficien o perjudiquen su desarrollo; es más, es posible que incluso reestructuren o suplanten el aprendizaje emocional aprendido en el hogar. Este entorno suele ser el escolar, razón que justifica el ámbito educativo para desarrollar la I.E. de un modo positivo (Gallego-Gil \& Gallego-Alarcón, 2006). 
No obstante, a pesar de que a lo largo de toda la vida la I.E. se pueda seguir desarrollando o modificando, los primeros años son cruciales, pues es cuando se forjan las habilidades emocionales y se asientan los rudimentos de la I.E. (Goleman, 1995). Además, según Henao y García (2009) es en la infancia cuando los niños son conscientes de sus emociones y cuando comienzan a ser capaces de reconocer las emociones de los demás y sus causas. Al comenzar la etapa de Educación Infantil los niños tienen bastante facilidad para adentrarse en el mundo de los sentimientos, puesto que están más conectados al mundo emocional que los adultos y les cuesta menos trabajo observarse a sí mismos (Boix, 2007). En este sentido, la función del educador no es solo promover el desarrollo de la I.E; también lo es procurar que los niños no pierdan, conforme van creciendo y avanzando en el sistema educativo, esa capacidad emocional con la que comienzan al principio de su escolarización que les permite no tener "miedo" ni "vergüenza" ante lo emocional y conectarse a este mundo con relativa facilidad.

Presuponiendo entonces la importancia del desarrollo de la I.E. desde los primeros años de vida, ¿por qué a lo largo de tanto tiempo no se ha tenido en cuenta? ¿Es compatible el desarrollo del cociente intelectual y el desarrollo de la I.E.? Según interesantes estudios realizados en esta línea, cabe afirmar que, más allá de ser compatibles, existe además un equilibrio entre lo racional y lo emocional, y esta relación es armoniosa, de tal manera que la habilidad intelectual incrementa (Freshwater \& Stickley, 2004).

Durante muchos años ha habido un error en la concepción del concepto "inteligencia emocional", y es que a menudo éste ha sido entendido como "ser amable" o "dar rienda suelta a nuestros sentimientos" (Goleman, 1998); sin embargo, tiene más bien que ver con la capacidad para explorar las propias emociones y controlarlas, motivarse a uno mismo, reconocer las emociones ajenas, y controlar las relaciones (Salovey \& Mayer, 1990). Tradicionalmente la formación correcta de la afectividad era vista como entorpecedora del conocimiento, y éste como lo único digno de importancia en educación (Valero, 2009). Durante muchos años, el principal objetivo académico ha sido el desarrollo del cociente intelectual ignorando el componente afectivo de la persona. Sin embargo, sabemos de personas con excelentes calificaciones académicas y un CI muy alto que carecen por completo de éxito en sus vidas y son incapaces de controlarlas (Goleman, 1995). De este modo, emociones e inteligencia han sido vistas con frecuencia como incompatibles o contrarias, puesto que se consideraba que las primeras perturbaban a las segundas (Mestre \& Fernández-Berrocal, 2007); no obstante, sabemos que en no pocas ocasiones ocurre justo al revés, ya que promueven el pensamiento al ayudar a centrar la atención o a superar las inadaptaciones. En estos casos podemos decir que las emociones, no solo no perturban la inteligencia, sino incluso la potencian.

En realidad, como se ha constatado, el cociente intelectual, a pesar de ser tan valorado, "en el mejor de los casos parece aportar tan solo un $20 \%$ de los factores determinantes del éxito" (Goleman, 1995, p. 64). El 80\% restante es lo que Daniel Goleman llama I.E. e incluye características como:

La capacidad de motivarnos a nosotros mismos, perseverar en el empeño a pesar de las posibles frustraciones, controlar los impulsos, diferir las gratificaciones, regular nuestros propios estados de ánimo, evitar que la angustia interfiera en nuestras facultades racionales, capacidad de empatizar y confiar en los demás (p. 65).

En síntesis, hoy en día, resulta incomprensible que existan escuelas en las que aún se insista en potenciar las habilidades académicas en detrimento de la I.E., esencialmente por dos motivos:

- Por una parte, si consideramos como una de las finalidades de la educación el desarrollo de la persona, es necesario potenciar la adaptación social del individuo: la I.E. es aquí fundamental (Mestre \& Fernández-Berrocal, 2007). $\mathrm{Si}$, además, hablamos de "la educación integral del sujeto", sin duda atañe al desarrollo de la I.E. (Gallego-Gil \& Gallego-Alarcón, 2006). 
- Por otra parte, los procesos de cognición y emoción son inseparables, puesto que las interacciones entre ambas son continuas. De hecho, la I.E. no se refiere solo a las emociones sino también a la cognición. Según Gallego-Gil y GallegoAlarcón (2006, p. 93) "las interrelaciones continuas que van de lo emocional a lo cognitivo y viceversa, son las que serían auténticamente características de la inteligencia emocional". Por tanto, es inútil reprimir el desarrollo involuntario de la I.E. en el aula.

Es posible, en este caso, decir que al desarrollo de la inteligencia académica o abstracta le es beneficioso el desarrollo de la I.E. Pues existe una relación circular entre ambas, y está confirmada empíricamente, según Mestre y Fernández-Berrocal (2007), la existencia de una asociación entre inteligencia emocional y académica. En esta línea, otros consolidados autores en este campo han evidenciado que los alumnos con un alto nivel de I.E. presentaban menores niveles de agotamiento, mayor eficacia académica y menor percepción de estrés, así como puntuaciones más elevadas en vigor, dedicación y absorción en el desempeño de sus tareas académicas (Extremera, Durán \& Rey, 2007).

En algunas ocasiones los aspectos emocionales son la etiología de los problemas escolares, pero en otros casos estos mismos aspectos emocionales pueden ser los que faciliten el aprendizaje. Según Goleman (1995), otros motivos por los que la I.E. se relaciona con el rendimiento académico tienen que ver con el pensamiento positivo o tener altas expectativas con respecto a lo que puedes conseguir. Por tanto, es posible utilizar las emociones para facilitar el aprendizaje (Denham, Zinsser \& Bailey, 2011). Los estudios de Shipley, Jackson y Segrest (2010) indican que las habilidades de la I.E. conducen a un rendimiento superior en las tareas escolares. Y, a medida que uno va alcanzando sus objetivos y logros académicos, también va tornándose emocionalmente más inteligente, teniendo más confianza en sí mismo y sabiendo automotivarse y controlarse más.

Podríamos concluir que el desarrollo de la I.E. es algo fundamental, tanto para favorecer el rendimiento académico, como para formar personas preparadas capaces de llevar a cabo una vida plena; no debemos entonces permitir que se produzca únicamente de modo involuntario e inconsciente, sin prestarle la importancia y dedicación que merece dentro de la planificación educativa, puesto que, en efecto, repercute en el bienestar individual y colectivo de todos.

\section{Estrategias para el desarrollo de la I.E. en el aula}

Una de las causas que justifican la poca relevancia que, en no pocas ocasiones se otorga a la educación emocional, proviene del desconocimiento y falta de formación de los propios maestros, quienes no siempre poseen herramientas ni recursos para ponerla en práctica (Denham et al., 2011). Por ello, consideramos fundamental divulgar y poner al alcance de quienes se dedican a esta tarea, determinadas estrategias dirigidas a potenciar el desarrollo de la I.E. en los alumnos, para que, efectivamente, constituya uno de los pilares básicos de nuestros diseños de programación en el aula.

Como estrategia principal, cabría destacar el desarrollo de la I.E. del docente, ya que "todas las personas que forman parte de un ámbito aportan a él su emocionalidad y de alguna manera son influidos por la emocionalidad del mismo" (Boix, 2007, p. 23). Por tanto, es preciso reconocer a los maestros como agentes activos del clima emocional del aula, siendo así fundamental que cada educador se trabaje emocionalmente a sí mismo, de manera que esté al mismo tiempo capacitado para intervenir en la educación emocional de sus alumnos.

Además, tal y como afirman Kremenitzer y Miller (2008), los niños aprenden por imitación, y por ello los maestros deben constituir un ejemplo positivo. Precisamente por este motivo es tan importante que se adquieran habilidades emocionales a través de programas de aprendizaje socioemocional, (aparte de los programas destinados a los alumnos). Del mismo modo, a medida que los educadores se implican, mayor es la empatía para entender, mirar y trabajar con los alumnos y percibir 
las necesidades de desarrollo que tienen, contribuyendo así a fomentar sus habilidades (Boix, 2007).

De acuerdo con Gallego-Gil y GallegoAlarcón (2006, p.193) "el desarrollo de la I.E. es un aprendizaje implícito o incidental que se genera aunque no seamos conscientes de que estamos aprendiendo ni tengamos el propósito deliberado de aprender", ya que todos los contenidos que se aprenden en el contexto escolar tienen un componente emocional. Indudablemente, no por ello debemos dejar de fomentar su desarrollo, ni dejar de preocuparnos por los procesos que, de manera explícita y programada, pueden mejorar sustancialmente su adquisición.

En esta línea, y según estos mismos autores, el centro puede intervenir en la educación emocional a través de tres ámbitos distintos. En primer lugar mediante la actuación diaria; aunque muchas veces sea de una forma involuntaria, los alumnos aprenden por medio de la observación, pues para ellos el maestro es su modelo. En segundo lugar, al programar, dentro del margen de los contenidos actitudinales y transversales. Y en tercer lugar, al responsabilizar a alguien en este tipo de enseñanza de un modo más concreto mediante programas específicos.

Muchos de los aspectos referidos al quehacer diario no pueden ser aquí detallados, puesto que la mayoría son inconscientes. A pesar de ello, algunos comportamientos cotidianos (el ser empáticos, receptivos, establecer relaciones cordiales y afectivas, ofrecerles oportunidades de mejorar, hacerles sentir comprendidos...) pueden tornarse más efectivos mediante la reflexión.

Con respecto al modo de programar, a menudo los maestros se muestran reacios con la idea de añadir nuevos temas al currículum; sin embargo, las emociones forman parte de toda la vida del niño y pueden y deben integrarse mediante los contenidos actitudinales y transversales. Esto les ayuda a comprender mejor las conexiones existentes entre el conocimiento académico y la experiencia de vida, e incluso a incorporar los conocimientos escolares a su propia experiencia vital (Centros Familiares de Enseñanza, 2001). A continuación se ofrecen algunas estrategias y recomendaciones que pueden servir de ayuda para la práctica docente, conscientes de que, a menudo, suele resultar costoso encontrar contenidos de tipo actitudinal para trabajar en el aula de forma consecuente y planificada.

- En Infantil, especialmente, se debe aprovechar la rutina de la asamblea para conversar con los niños y que expresen sus emociones, aunque para ello es necesario haber creado un clima de respeto, relajación y confianza en el que los alumnos estén cómodos expresándose (Boix, 2007). La relajación es una de las técnicas más utilizadas, por lo que es fundamental concienciar sobre el beneficio de la respiración y la distensión de músculos desde una edad temprana para ser más tarde capaces de transferir este aprendizaje a distintas situaciones (Ribes, Bisquerra, Agulló, Filella \& Soldevila, 2005).

- Cada vez que se identifique una emoción en un alumno es conveniente ponerle nombre (enfado, alegría, miedo...). Una estrategia interesante en este sentido puede ser animar a los niños a "dibujar caras" donde aparezcan emociones básicas (alegría, enfado, miedo, tristeza, sorpresa...). Si animamos al niño a hablar sobre ellas y hacer partícipe al resto del grupo en la identificación de las distintas expresiones faciales, estaremos promoviendo la empatía y la aceptación de que todos, en algún momento, podemos llegar a sentirnos así.

- Por otra parte, para que los niños vayan aprendiendo a controlar sus emociones también deben conocer cuál es el origen de las mismas (González-Ramírez, 2007), ello les ayudará a establecer relaciones adecuadas entre pensamiento, emoción y comportamiento. Es importante que reflexionen sobre sus propias reacciones y comprendan cómo, ante un mismo hecho, las personas pueden comportarse de manera distinta sin que por ello hayan de ser juzgadas.

- En este sentido, les puede animar el hecho de que el maestro comente en voz 
alta sus propias emociones ("qué triste me pongo cuando os peleáis", "qué contenta estoy cuando compartís"...). Poner ejemplos concretos que nos ayuden a expresar nuestros sentimientos y reacciones, o utilizar relatos breves para ilustrarlo, pueden ser estrategias muy apropiadas para lograrlo.

- Al hablar con los niños sería más coherente utilizar el verbo "estar" en vez de "ser", pues es más constructivo utilizar un verbo transitorio que deja abierta la opción de cambio (Boix, 2007). De esta manera, se sienten más capaces de transmutar sus emociones, ya que no se trata de eliminar las emociones negativas sino de transformarlas en positivas, por ejemplo pasando de estar tristes a estar alegres. Para ello podemos plantearles distintas expresiones y que ellos identifiquen qué sentimiento hay tras ellas y de qué forma podemos (re)convertirlas. Que verbalicen y manifiesten cómo identificarlas $\mathrm{y}$ transformarlas.

- Es importante que sepan cuándo se han equivocado, siempre enseñándoles que es posible mejorar, de forma que se relacionen con el error y éste también contribuya a su aprendizaje (GonzálezRamírez, 2007). Re(conocer) las propias fortalezas y debilidades puede ser un primer paso para poder modificar todo aquello que nos hace sentir mal. Así por ejemplo, si identificamos las emociones con los sentidos, podríamos ayudarles a definir cómo identificarlas y expresarlas al objeto de conocernos y expresarnos mejor.

- Evidentemente, fomentar las habilidades sociales a través del trabajo en equipo, los encuentros interpersonales y la mejora de la comunicación, contribuye al desarrollo de su I.E. (GonzálezRamírez, 2007). El teatro y el relato de cuentos o acontecimientos de la vida cotidiana, son estrategias muy adecuadas para trabajar en esta dirección.

- Por ello la distribución de roles, el reparto de funciones... también favorece la socialización y posibilita la autoestima al sentirse integrado, aceptado e incluso a veces protagonista entre sus compañeros (Gallego-Gil \& Gallego-Alarcón, 2006).

- A través de las lecturas y de los sentimientos de los distintos personajes que aparecen en ellas es posible trabajar los sentimientos de los niños, la empatía, el compañerismo y la solidaridad entre otros valores (Mestre \& FernándezBerrocal, 2007).

- Plantear problemas, preguntar y debatir acerca de sus posibles soluciones (Mayer \& Caruso, 2000), puede ayudar a controlar las emociones cuando abordemos una situación similar.

- Para liberar sus emociones, y progresivamente ir atenuándolas sin reprimirlas, la práctica psicomotriz puede ser muy útil (Aucouturier, 2012). La psicomotricidad puede contribuir a sentirnos más libres, experimentando nuevas sensaciones que nos hacen conocernos mejor, adquirir mejor conciencia de nosotros mismos $y$ expresarnos tal como nos sentimos. E1 lenguaje corporal, en definitiva, refuerza la seguridad emocional, con especial énfasis en las edades más tempranas.

En cuanto a programas específicos elaborados para desarrollar la I.E, se exponen algunas actividades extraídas de un programa destinado a niños de la etapa de Educación Infantil, distribuidas en cinco bloques temáticos (López-Cassà, 2007).

El primer bloque temático es "La conciencia emocional", pues el niño debe ser capaz de identificar, reconocer y expresar sus propias emociones y tomar conciencia de que los demás también tienen emociones y las expresan. Una de las actividades dentro de este bloque es: "Cómo se sienten". En esta actividad, utilizaremos tarjetas con dibujos de caras que expresan distintos sentimientos (alegría, tristeza, enfado...), los niños deben reconocer la emoción, describir la imagen y expresar cuándo se sienten ellos así. Otra actividad es "Mi cara refleja". En ella, se situarán delante del espejo y deberán ir poniendo las distintas expresiones faciales que indique el maestro. 
En "La varita mágica", habrá un saco con tarjetas con distintas expresiones faciales y al hacer "magia" con la varita, la cara de los niños tendrá la misma expresión que la que se refleja en la tarjeta escogida.

"Regulación emocional": es el segundo bloque, pues los niños deben aprender a transformar las emociones negativas, que nos hacen tener comportamientos poco saludables, en emociones positivas. Una de las actividades que podemos encontrar es "¡Qué tranquilidad!", en la que se hará una relajación con los niños tumbados en el suelo, de manera que el tono muscular no esté rígido, concentrándonos en distintas partes del cuerpo (brazos, piernas y cara). Otras actividades son "¿Qué puedo hacer cuando me sienta triste?”, “¿Qué puedo hacer cuando me sienta enfadado?" y "¿Qué puedo hacer cuando me sienta asustado?; a partir de cuentos y de las distintas emociones que experimentan los personajes que aparecen, les preguntaremos a los niños si ellos alguna vez se han sentido así, qué hacen cuando les ocurre y aportarán entre todos distintas soluciones para sentirse mejor.

El tercer bloque es "Autoestima", ya que los niños deben ser capaces de conocer, valorar y aceptar sus capacidades y limitaciones sin dejar de quererse a sí mismos. Algunas de las actividades para ello son: "Me quieren", en la que a partir del cuento "El Patito Feo", adaptado para la ocasión, trabajarán sus propias características personales. Después de contar el cuento haremos preguntas sobre el mismo: "¿cómo era?, ¿cómo le llamaban?, ¿cómo se sentía?, ¿qué le pasó?, ¿acabó siendo feliz? ¿era un pato?; tras esto reflexionaremos acerca de las personas que les quieren y las cosas que les gustan de ellos. La siguiente actividad es "Soy el rey": cada semana le tocará a un niño llevar una corona y "ser el rey" gozando de "privilegios" como elegir el cuento que quieren leer, cantar su canción favorita, elegir un juego... Además, las familias escribirán una carta con sus cualidades y ésta será leída en clase. Cuando termine la semana se reflexionará acerca de cómo se ha sentido, qué le ha gustado y el resto de compañeros dirá una cualidad de este niño.
"Habilidades socio-emocionales", para fomentar el desarrollo de competencias que faciliten las relaciones interpersonales, es el cuarto bloque. Las actividades son: "iQuiero ser el primero en la fila!": mediante un teatro se representará una historia con un situación muy próxima a ellos, el de querer ser el primero en la fila. En el cuento se da un conflicto en el que dos niños se pelean por ser los primeros en la fila; para solucionarlo, la maestra utilizará una lista con los nombres de todos los niños de la clase y que tengan la misma oportunidad de ser los primeros estableciendo turnos. En la actividad "Se me ha roto", a través de imágenes se muestra cómo un personaje rompe sin querer una página de un libro, y acude muy preocupado a su maestra, quien le ayuda a arreglarlo y le felicita por haber contado la verdad. Después, a través de preguntas, reflexionaremos sobre cómo se sentía el personaje y qué hubiésemos hecho nosotros en la misma situación.

El último bloque es "Habilidades de vida" pues los niños deben identificar los distintos sentimientos que podemos experimentar en cada uno de los entornos que abarca nuestra vida. Una actividad es "Gorros de colores": utilizaremos un gorro rojo y uno verde para identificar aquellas situaciones que pueden ser molestas o agradables (respectivamente) en nuestra vida cotidiana. El maestro irá indicando distintos momentos y en función de si piensan que son agradables o desagradables escogerán un gorro $\mathrm{u}$ otro $\mathrm{y}$ se lo pondrán explicando cómo se sienten.

Estos son solo algunos ejemplos generales de actividades y actuaciones docentes que hemos querido destacar y que pueden llevarse a cabo en el aula para fomentar el desarrollo de la I.E. Dependiendo de la realidad escolar, el contexto, el equipo pedagógico, los proyectos educativos...las estrategias más eficaces para el desarrollo de la I.E. pueden ser muy distintas (Gómez-Bruguera, 2003). Por supuesto, no hay nada universal ni infalible, por ello es tan importante conocer las características del alumnado, del centro y del entorno en el que desempeñamos nuestra labor educativa. Lo cierto es que se ha demostrado que los niños que aprenden habilidades sociales y emocionales tienen mayor éxito, no solo en la escuela, sino en todas las áreas de la vida (Lantieri, 2010). 


\section{Conclusiones y propuestas educativas}

Retomando los objetivos expuestos, se subraya que la I.E. es un proceso natural que puede desarrollarse a lo largo de toda la vida, aunque los primeros años son especialmente importantes, así como la influencia del ámbito familiar y escolar.

Durante la etapa de la Educación Infantil es cuando se desarrollan las habilidades emocionales y cuando más abiertos estamos al mundo emocional. En años posteriores será posible seguir forjando la I.E., pero su educación debe darse durante los primeros años, pues más adelante podría resultar complejo.

El hecho de ser un proceso natural no es óbice para que podamos trabajarlo de un modo consciente y potenciar esta inteligencia al objeto de destacarla lo máximo en beneficio del rendimiento académico y del propio éxito, bienestar y felicidad personal de cada individuo. Un beneficio que antiguamente se le atribuía al nivel de cociente intelectual de las personas y que no puede vincularse exclusivamente a ella sin reconocer la extraordinaria influencia que tiene en la vida de las personas.

A lo largo de todo el trabajo se ha hecho hincapié en la importancia de la I.E. y la necesidad de llevar a la práctica determinadas estrategias para fomentar su desarrollo. Sin embargo, seguramente los "buenos maestros", (entendiendo por "buenos maestros", no aquellos que realizan su labor a la perfección, sino aquellos que, siendo conscientes de sus errores, buscan mejorar cada día y se preocupan y desviven por el bienestar y felicidad de sus alumnos), antes de que se popularizase el concepto, de una forma innata ya llevaban a la práctica actuaciones docentes encaminadas al reconocimiento y control de las propias emociones, reconocimiento de las emociones ajenas, automotivación y relaciones interpersonales, es decir actuaciones que promovían el desarrollo de la I.E. aunque aún no aludiesen a ella como tal.

Hoy en día se (re)conoce el nombre de esta inteligencia, y también se diferencia y se es capaz de poner nombre a todas las actuaciones docentes a través de las cuales el maestro puede trabajar la educación emocional de sus alumnos. Estas son: el quehacer diario, los contenidos actitudinales o transversales y los programas específicos. Por tanto, actualmente se sabe que la educación emocional está presente en las escuelas, pero además podemos descubrir qué nivel puede alcanzar a través de las diferentes actuaciones encaminadas a su logro. Existen al respecto diversos estudios que evidencian la existencia de un conjunto de habilidades emocionales interrelacionadas que los programas de aprendizaje socioemocional en el aula deberían recoger y enfatizar, tales como atender a los sentimientos, comprender las emociones y usarlas en nuestro razonamiento para solucionar problemas y aprender a regular los estados afectivos propios y ajenos (Extremera \& Fernández-Berrocal, 2003), aunque no es menos cierto que resta mucho camino por recorrer.

Para finalizar, cabe decir que, aun considerando la I.E. y la inteligencia cognitiva como compatibles, así como las distintas y múltiples interrelaciones entre ambas, es preciso reconocerlas como inseparables, ya que la I.E. no abarca solo las emociones en sí, independientemente de cómo se utilicen o de la conciencia que se tenga acerca de las mismas, sino que atañe al hecho de ser emocionalmente inteligentes e inteligentemente emocionales.

No obstante, es preciso hacer las siguientes consideraciones:

- En primer lugar, no siempre la I.E. se trabaja a través de programas específicos de educación emocional debido a las limitaciones con las que se encuentran algunos maestros. A menudo, tienen que seguir un currículum o programaciones demasiado estrictas, lo que les impide realizar actividades concretas destinadas a su desarrollo. En ocasiones, esta misma preocupación por alcanzar todos los objetivos y competencias que se les exigen es la que hace que se olviden de trabajar la educación emocional, al menos desde los contenidos transversales y actitudinales. Por tanto, sería preciso diseñar un currículum y una programación más flexibles, al tiempo que priorizar determinados contenidos, teniendo en cuenta la influencia de 
la I.E. en el éxito presente y futuro de nuestros alumnos.

- En segundo lugar, muchas veces la I.E. no solo no se trabaja sino que las repercusiones se desconocen, puesto que es un tema sobre el que los docentes apenas tienen preparación. Por ello, se torna imprescindible incluir la I.E. como un contenido fundamental dentro de los planes de formación de los futuros docentes en la Facultad, para que las nuevas promociones de maestros estén mejor formados en esta materia y de este modo se muestren más interesados y capacitados para trabajar. Dicha formación deberá incluir también programas destinados al desarrollo de la I.E. de los propios estudiantes universitarios. Porque tanto la conciencia emocional, como la regulación y autonomía emocional, las competencias sociales y las habilidades de vida y bienestar, pueden enseñarse y aprenderse (Pérez-Escoda, Filella, Alegre \& Bisquerra, 2012).

- En tercer lugar, muchos maestros no trabajan la I.E. porque ello implica un esfuerzo que, en la actualidad, no se encuentra recogido ni reconocido, formando parte del "currículum oculto"; por consiguiente, no sienten la necesidad, ni contemplan la recomendación de promoverla. Sin embargo, forma parte de sus requerimientos como educadores $\mathrm{y}$, por supuesto, es su responsabilidad ya que, como decíamos, el maestro debe velar por el desarrollo integral de la persona. En este sentido, otra propuesta interesante es motivarlos $\mathrm{y}$ hacerles conscientes de la importancia de su trabajo, así como diseñar pautas en la elección de carrera para procurar que quienes elijan esta profesión lo hagan impulsados por su vocación.

La labor del maestro no se resume en la trasmisión o enseñanza de contenido teórico, va mucho más allá. La labor del maestro no es educar "por partes", de manera segmentada, sino abarcar a la persona en su totalidad. En este sentido, diversos estudios han mostrado cómo el rol del educador o educadora de la primera infancia ha de centrarse en un enfoque holístico e integral, de manera que el enfoque afectivo incida en el desarrollo de habilidades para la vida y haga partícipes de dicho proceso a toda la comunidad educativa (Zapata \& Ceballos, 2010).

El "buen maestro" se vale de la I.E. para que su enseñanza no sea solo un aprendizaje escolar, sino más bien suponga un aprendizaje para la vida, reconociendo que las emociones nos acompañan y abarcan por completo. Es preciso así dar sentido a cada una de nuestras experiencias, puesto que, tal y como afirma Valero (2009, p. 55): "la inteligencia emocional es a la vida lo que la sal a los alimentos".

\section{Lista de referencias}

Aucouturier, B. (2012). L'enfant terrible. ¿Qué hacer con el niño difícil en la escuela? Barcelona: Graó.

Birknerová, Z., Frankovský, M. \& Zbihlejová, L. (2013). Social Intelligence in the Context of Personality Traits of Teachers. American International Journal of Contemporary Research, 3 (7), pp. 11-17.

Bisquerra, R. (2003). Educación Emocional y competencias básicas para la vida. Revista de Investigación Educativa, 21 (1), pp. 7-43.

Boix, C. (2007). Educar para ser feliz. Una propuesta de educación emocional. Barcelona: Ceac.

Buitrago, R. E. (2012). Contexto escolar e inteligencia emocional en instituciones educativas públicas del ámbito rural y urbano del departamento de Boyacá (Colombia). Tesis doctoral. Universidad de Granada. Granada, España.

Castro, P. J., Van der Veer, R., Burgos-Troncoso, G., Meneses-Pizarro, L., Pumarino-Cuevas, N. \& Tello-Viorklumds, C. (2013). Teorías subjetivas en libros latinoamericanos de crianza, acerca de la educación emocional. Revista Latinoamericana de Ciencias Sociales, NiñezyJuventud, 11(2), pp. 703-718. Doi:10.11600/1692715x.11217060213.

Centros Familiares de Enseñanza (2001). La Inteligencia Emocional. Una Brújula para 
el siglo XXI. III Jornadas de innovación pedagógica. Granada: Autor.

Denham, S. A., Zinsser K. \& Bailey, C. S. (2011). Emotional Intelligence in the First Five Years of Life. Encyclopedia on Early Childhood Development, 1, pp. 1-7.

Duer, C. (2010). Infancia, desarrollo y conocimiento: los niños y niñas y su socialización. Revista Latinoamericana de Ciencias Sociales, Niñez y Juventud, 8 (2), pp. 799-808.

Dumbrava, G. (2011). Workplace relations and emotional intelligence. Annals of the University of Petroşani, Economics, 11 (3), pp. 85-92.

Durán, S. (2013). Los rostros y las huellas del juego: creencias sobre el juego en la práctica docente del profesorado en dos centros infantiles de la Secretaría Distrital de Integración Social (SDIS), en Bogotá,D.C.-Colombia. Tesis Doctoral. Universidad de Granada, Granada, España.

Extremera, N. \& Fernández-Berrocal, P. (2003). La inteligencia emocional en el contexto educativo: hallazgos científicos de sus efectos en el aula. Revista de Educación, 332, pp. 97-116.

Extremera, N., Durán, A. \& Rey, L. (2007). Inteligencia emocional y su relación con los niveles de burnout, engagement y estrés en estudiantes universitarios. Revista de Educación, 342, pp. 239-256.

Freshwater, D. \& Stickley, T. (2004). The heart of the art: emotional intelligence in nurse education. Nursing Inquiry, 11 (2), pp. 91-98. Doi: 10.1111/j.1440-1800.2004.00198.x

Gallego-Gil, D. J. \& Gallego-Alarcón, M. J. (2006). Educar la inteligencia emocional en el aula. Madrid: PPC.

Gardner, H. (1993). Frames of Mind: The Theory of multiple intelligences. New York: Basic Books.

Goleman, D. (1995). Inteligencia Emocional. Barcelona: Kairós.

Goleman, D. (1998). La práctica de la Inteligencia emocional. Barcelona: Kairós.
Gómez-Bruguera, J. (2003). Educación emocional y lenguaje en la escuela. Barcelona: Octaedro.

González-Ramírez, J. F. (2007). Inteligencia emocional. La manera de manejar las emociones. Madrid: Platinum Selecta.

Henao, G. \& García, M. C. (2009). Interacción familiar y desarrollo emocional en niños y niñas. Revista Latinoamericana de Ciencias Sociales, Niñez y Juventud, 7 (2), pp. 761-783.

Kremenitzer, J. P. \& Miller, R. (2008). Are You a Highly Qualified, Emotionally Intelligent Early Childhood Educator? Young Children, 63 (4), pp. 106-112.

Lantieri, L. (2010). Las emociones van a la escuela. New York: National Geographic,

López-Cassà, E. (2005). La educación emocional en la educación infantil. Revista Interuniversitaria de Formación del Profesorado, 19 (3), pp. 153-167.

López-Cassà, E. (2007). Educación emocional. Programa para 3-6 años. Educación emocional y en valores. Madrid: Wolters Kluver.

Marina, J. A. (2005). Precisiones sobre la educación emocional. Revista Interuniversitaria de Formación del Profesorado, 19 (3), pp. 27-43.

Marrodán, A. (2013). Inteligencia Emocional. Psicología, Educación, Salud y Vida. Recuperado de:

http://asunmarrodan.wordpress.com/ inteligencia-emocional/

Mayer, J. D. \& Caruso D. R. (2000). Traditional Intelligence Meets Traditional Standarts for an Intelligence. Intelligence 27 (4), pp. 267-298.

Mayer, J. D., DiPaolo, M. T. \& Salovey, P. (1990). Perceiving affective content in ambiguous visual stimuli: A component of emotional intelligence. Journal of personality Assessment, 54, pp. 772-781.

Mestre, J. M. \& Fernández-Berrocal, P. (2007). Manual de la inteligencia emocional. Madrid: Pirámide.

Mikulic, I. Mª (2013). La educación emocional y social en Argentina: entre certezas y esperanzas. En C. Clouder (coord.) Educación Emocional y Social. Análisis 
Internacional. Santander: Fundación Botín.

Payton, J., Weissberg, R. P., Durlak, J. A., Dymnicki, A. B., Taylor, R. D., Schellinger, K. B. \& Pachan, M. (2008). The Positive Impact of Social and Emotional Learning for Kindergarten to Eighth-Grade Students. Findings from Three Scientific Reviews. Chicago: Collaborative for Academic, Social and Emotional Learning.

Pena, M. \& Reppeto, E. (2008). Estado de la investigación en España sobre Inteligencia Emocional en el ámbito educativo. Revista electrónica de investigación psicoeducativa, 6 (15), pp. 401-420.

Pérez-Escoda, N., Filella, G., Alegre A. \& Bisquerra, R. (2012). Desarrollo de la competencia emocional de maestros y alumnos en contextos escolares. Electronic Journal of Research in Educational Psychology, 10 (3), pp. 1183-1208.

Ribes, R., Bisquerra, R., Agulló, M. J., Filella, G. \& Soldevila, A. (2005). Una propuesta de currículum emocional en educación infantil (3-6 años). Cultura y Educación: Revista de teoría, investigación y práctica, 17 (1), pp. 5-18.

Rodríguez-Sánchez, R. A. (2000). La expresión de las emociones en Charles Darwin. Educación de valores y valores para la educación. Emociones. Thémata, 25, pp. 335-344.

Salovey, P. \& Mayer, J. D. (1990). Emotional Intelligence. Imagination, Cognition $y$ Personality, 9, pp. 185-211.

Shipley, N. L., Jackson, M. J. \& Segrest, S. L. (2010). The effects of emotional intelligence, age, work experience and academic performance. Research in Higher Education Journal, (9), pp. 1-18.

Valero, J. M. (2009). ¿Analfabetos emocionales? Educar los sentimientos en la escuela. Estrategias educativas. Madrid: Instituto Calasanz de Ciencias de la Educación (ICCE).

Zapata, B. E. \& Ceballos, L. (2010). Opinión sobre el rol y perfil del educador para la primera infancia. Revista Latinoamericana de Ciencias Sociales, Niñez y Juventud, 8 (2), pp. 1069-1082. 